\title{
Experimental Evolution of Olfactory Memory in Drosophila melanogaster
}

\author{
Frederic Mery ${ }^{1, *, \dagger}$ \\ Juliette Pont ${ }^{1, \star}$ \\ Thomas Preat ${ }^{2}$ \\ Tadeusz J. Kawecki ${ }^{1, *}$ \\ ${ }^{1}$ Department of Biology, University of Fribourg, Chemin du \\ Musée 10, CH-1700 Fribourg, Switzerland; ${ }^{2}$ Gènes et \\ Dynamique des Systèmes de Mémoire, Centre National de la \\ Recherche Scientifique, Ecole Supérieure de Physique et de \\ Chimie Industrielles, Paris, France
}

Accepted 1/30/2007; Electronically Published 5/7/2007

\begin{abstract}
In order to address the nature of genetic variation in learning performance, we investigated the response to classical olfactory conditioning in "high-learning" Drosophila melanogaster lines previously subject to selection for the ability to learn an association between the flavor of an oviposition medium and bitter taste. In a T-maze choice test, the seven high-learning lines were better at avoiding an odor previously associated with aversive mechanical shock than were five unselected "low-learning" lines originating from the same natural population. Thus, the evolved improvement in learning ability of high-learning lines generalized to another aversion learning task involving a different aversive stimulus (shock instead of bitter taste) and a different behavioral context than that used to impose selection. In this olfactory shock task, the high-learning lines showed improvements in the learning rate as well as in two forms of consolidated memory: anesthesia-resistant memory and longterm memory. Thus, genetic variation underlying the experimental evolution of learning performance in the high-learning lines affected several phases of memory formation in the course of olfactory aversive learning. However, the two forms of consolidated memory were negatively correlated among replicate high-learning lines, which is consistent with a recent hypothesis that these two forms of consolidated memory are antagonistic.
\end{abstract}

\footnotetext{
* These authors contributed equally to this work.

${ }^{\dagger}$ Present address: Laboratory for Evolution, Genome, and Speciation, Centre National de la Recherche Scientifique, Gif sur Yvette, France.

${ }^{\ddagger}$ Corresponding author; e-mail: tadeusz.kawecki@unifr.ch.
}

Physiological and Biochemical Zoology 80(4):399-405. 2007. (C) 2007 by The University of Chicago. All rights reserved. 1522-2152/2007/8004-0128\$15.00

\section{Introduction}

Learning allows an animal to develop within its lifetime an adaptive response to novel situations, even those never encountered in the evolutionary past of the species. The ability to learn is thus among the greatest achievements of organic evolution and a trait of particular interest for evolutionary biology. Yet our understanding of the evolutionary biology of learning is rudimentary; in particular, we know next to nothing about the nature of genetic variation affecting learning ability (Dukas 2004). We have been conducting an experimental evolution study in which replicated populations of Drosophila melanogaster ("high-learning" lines) are subject to selection for improved learning in the context of oviposition substrate choice (Mery and Kawecki 2002). That selection regime favored flies that associate the flavor of an oviposition substrate (orange or pineapple) with the bitter taste of quinine and avoid oviposition on this substrate several hours later, even though quinine is no longer present. Within 20 generations of selection, the selected high-learning lines evolved a markedly improved performance in this learning assay compared to the unselected controls ("low-learning" lines). This improvement was due to both faster learning and longer memory but not due to better discrimination or detection of the stimuli (Mery and Kawecki 2002). However, this evolutionary response was associated with costs: the high-learning lines showed poorer larval competitive ability (Mery and Kawecki 2003) and a reduction in fecundity when repeatedly forced to learn under nutritional stress (Mery and Kawecki 2004).

The response of the high-learning lines to selection was probably based on the genetic variation already present in the natural population, from which those lines were derived shortly before selection commenced. The results presented in this article address two questions concerning the nature of this response and thus the underlying genetic variation.

First, our high-learning lines were subject to selection for associative aversive learning in the context of particular stimuli (orange, pineapple, quinine), a particular behavior (oviposition), and a particular context (the oviposition cages; Mery and Kawecki 2002). To what extent does their improved learning performance generalize to other stimuli, tasks, and contexts? This is relevant to the general question, To what extent does evolution target general aspects of learning processes (a "general intelligence") as opposed to specialized learning skills specific to particular stimuli, behavioral tasks, and environmental contexts? This issue has been hotly debated in cognitive ecology 
(Healy and Braithwaite 2000; Macphail and Bolhuis 2001). To address this question we assayed the high- and low-learning lines in a learning test involving classical conditioning, in which the flies were conditioned to associate an airborne odor with mechanical shock and subsequently were tested for odor choice in a T-maze.

The second question is, Which forms of memory improved in the course of experimental evolution? Memory formation and consolidation consist of several distinct processes, some sequential and some apparently parallel. Studies on Drosophila (reviewed in Dubnau and Tully 1998; Wadell and Quinn 2001; Davis 2005) reveal four distinct forms of olfactory memory in flies. Short-term memory (STM) forms within seconds and decays within less than an hour, replaced by middle-term memory (MTM), which arises within minutes, reaches a peak at about an hour, and decays within several hours. Both STM and MTM are labile and easily erased by cold shock. Two other forms of memory-so-called anesthesia-resistant memory (ARM) and long-term memory (LTM) - are more resistant to those disturbances and are referred to as "consolidated." ARM begins to form within $30 \mathrm{~min}$ of conditioning and can persist for at least $24 \mathrm{~h}$. LTM is thought to begin forming within several hours of conditioning and can persist for days. LTM is protein synthesis-dependent, and its formation can be blocked by pharmacological protein synthesis inhibition, which has little effect on the other forms of memory. Furthermore, in classical conditioning, LTM forms only after several repeated conditioning trials separated by intervals (usually 15-30 min, the so-called spaced training protocol). Similar conditioning trials carried out immediately one after another (so-called massed protocol) result not in significant LTM but in ARM. ARM also forms after a single training cycle. Four analogous forms ("phases") of memory seem to occur in honeybees (Menzel 1999), while the mammalian model assumes three forms of memory (Dezazzo and Tully 1995). The existence of mutants and pharmacological interventions that selectively impair one form of memory with little effect on the others confirms that the memory forms are mechanistically distinct; the relationship among them is under debate (Tully et al. 1994; Isabel et al. 2004; Margulies et al. 2005). Even less is known about their evolutionary significance and dynamics, for example, whether evolution may act independently on different memory forms or which aspects of the process would change as a result of natural selection for improved learning performance. Here, by varying the conditioning protocol and the time of testing in the olfactory-mechanical shock assay, and by using cold shock to erase labile forms of memory, we attempt to "dissect" the stages of memory formation in the high- and low-learning lines. This allows us to infer which forms of memory changed in the course of the experimental evolution.

\section{Material and Methods}

\section{Selection Regime and Lines}

Seven selected high-learning lines (lines 1, 2, 3, 5, 6, 7, 8) and five unselected low-learning control lines (lines 1, 2, 4, 5, 7; Mery and Kawecki 2002) were used in this experiment. The origin of those lines and the regime under which they evolved are described in detail by Mery and Kawecki (2002). Briefly, all lines originated from the same base population derived from the field. The selection regime involved a choice between two flavors of oviposition media (orange and pineapple). It favored flies that learned an association between the flavor of medium and the aversive taste of quinine. Flies that remembered which of the two media had previously contained quinine and continued to oviposit on the other medium even though quinine was no longer present contributed more offspring to the next generation. The low-learning lines were not selected for learning but were otherwise maintained under the same conditions. All flies were bred on a standard cornmeal medium; the population was maintained at the size of about 150 adults. By the time of the experiments described below, the flies had been subject to selection for more than 100 generations. The response to selection within the first 47 generations is analyzed by Mery and Kawecki (2002). Since then, the lines continued to respond to selection, albeit with a slow rate: the proportion of eggs laid on the "correct" medium (i.e., the one that did not previously contain quinine) increased between generations 48 and 110 with the average rate of 0.0016 per generation.

\section{Olfactory Aversive Conditioning}

This procedure followed that of Mery and Kawecki (2005). Conditioning and tests were performed on samples of 50-70 flies (sexes mixed) raised at $25^{\circ} \mathrm{C}$ on standard cornmeal medium at a density of 200 eggs $/ 25 \mathrm{~mL}$ of food and aged 3-6 d from eclosion. These groups were isolated from culture bottles 3-5 h before conditioning, placed in empty vials, and kept at $25^{\circ} \mathrm{C}$ and $70 \%$ humidity until conditioning. We used 3-octanol and 4-methylcyclohexanol $(\mathrm{MCH})$ diluted in paraffin oil $(0.6$ $\mathrm{mL} / \mathrm{L}$ ) as odorants. The odors were delivered into the vial containing the flies with an air pump. The conditioning procedure consisted of one or several conditioning cycles. In each conditioning cycle (Fig. 1a), one odorant (CS+) was first delivered to the flies for $30 \mathrm{~s}$ accompanied by mechanical shocks (vibration) delivered for $1 \mathrm{~s}$ every $5 \mathrm{~s}$ by a test tube shaker (Heidolph Reax, Merck; 2,000 rpm). This period was followed by a 60-s rest period during which flies received humid air flow (no odor) and no shocks. For the subsequent $30 \mathrm{~s}$ the other odorant ( $\mathrm{CS}-$ ) was delivered without shocks. A conditioning cycle ended with a second rest period of $60 \mathrm{~s}$. Consecutive training cycles either followed one another immediately (massed protocol) or were separated by 20 -min rest intervals (spaced protocol). At a set time after the last conditioning cycle, 


\section{(a) Conditioning}

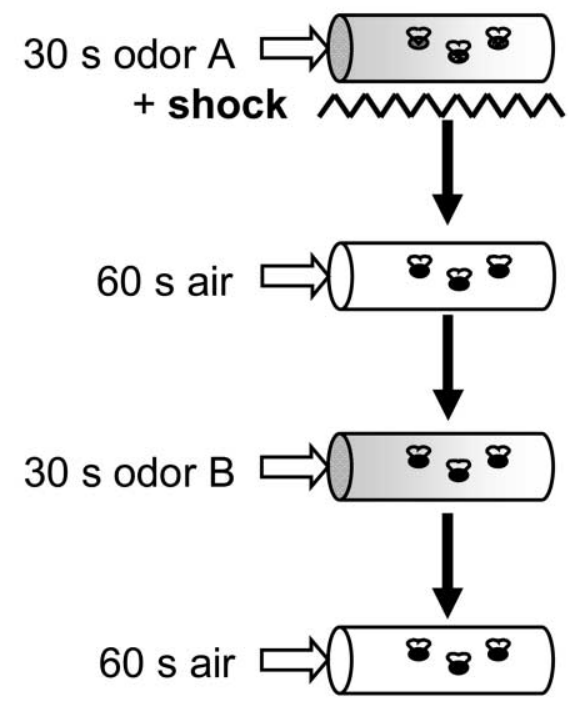

\section{(b) Test}

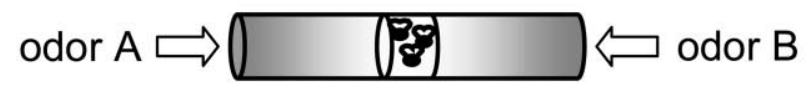

Figure 1. Aversive olfactory learning assay used in this article. $a$, Conditioning cycle. Multiple conditioning cycles were separated by 20 -min intervals (spaced protocol), or they followed one another immediately (massed protocol). $b$, Test. Flies were placed in a central chamber of a T-maze, where air currents carrying the two odors converged.

the flies were tested in complete darkness in a T-maze (Fig. 1b). The two odors were simultaneously presented, and the flies were permitted to move freely for $1 \mathrm{~min}$; the number of flies in each arm of the maze was subsequently counted. The proportion of flies that had moved toward octanol as opposed to $\mathrm{MCH}$ was then calculated; flies that remained in the central chamber of the maze were excluded from this calculation.

The design was counterbalanced so that each group of flies conditioned to avoid $\mathrm{MCH}$ was paired with another group from the same line that was conditioned to avoid octanol. The two paired samples were tested within $5 \mathrm{~min}$ of each other. For each pair of samples, a single value of memory score was calculated as the difference in the proportion of flies choosing octanol between the sample conditioned to avoid $\mathrm{MCH}$ and the sample conditioned to avoid octanol (maximum memory score is 1 ; a score of 0 means no response to conditioning). For the analysis (but not for graphical representation of the results), these proportions were arcsine-square root transformed (Sokal and Rohlf 1995).

\section{Memory Dissection}

In order to dissect the different memory phases of high-learning and low-learning lines, we performed the following five memory assays based on the olfactory aversive conditioning (for the logic behind these assays, see Tully et al. 1994; Isabel et al. 2004; Mery and Kawecki 2005):

1. A single conditioning cycle; test after $20 \mathrm{~min}$.

2. Massed protocol, three conditioning cycles; test after 20 min. The response in those two assays is mostly based on STM; the difference in response between them reflects the rate of learning acquisition.

3. Massed protocol, five conditioning cycles; test after $2.5 \mathrm{~h}$. The response in this assay is mainly based on MTM and ARM.

4. Massed protocol, five conditioning cycles, a cold shock at $2 \mathrm{~h}$ after the end of conditioning; test at $2.5 \mathrm{~h}$ after conditioning. The cold shock was applied by transferring the flies into a vial kept at $0^{\circ} \mathrm{C}$ in a bowl containing ice. After $2 \mathrm{~min}$, flies were transferred back to their original vial and were given $30 \mathrm{~min}$ to recover before being tested. The cold shock eliminates the labile forms of memory, so the response is based on ARM.

5. Spaced protocol, five conditioning cycles spaced at 20min intervals; test after $24 \mathrm{~h}$. The response in this assay would be mostly based on LTM.

For each memory assay we compared the memory score between high-learning and low-learning lines using a nested ANOVA (lines nested within selection regime). Because tests for each assay were carried out over several days, we also introduced "day as block" in the analysis. Assays 4 and 5 were carried out in two separate experiments six generations apart (generations 100 and 106); their results were analyzed both jointly and separately. The analyses were carried out with procedure GLM of the SAS 8.02 statistical package. The models were fitted using Type III sum of squares. Line and block were treated as random effects; the F-tests were obtained with statement RANDOM option TEST (SAS Institute 1989).

The results suggested a pattern of covariance between the memory scores in assays 4 and 5 across the high-learning lines, observed at both generation 100 and generation 106. To analyze this pattern, we used procedure MIXED of SAS (Littell et al. 1996) to estimate the variance-covariance matrix among and within the lines. This was done by using the mean memory scores per line and generation as data and fitting the following general mixed model:

$$
y_{i j k}=\mu_{j k}+g_{i j}+e_{i j k}
$$

where $y_{i j k}$ is the mean memory score of $i$ th line in $j$ th assay (assay 4 or 5) at generation $k, \mu_{j k}$ is the overall mean memory score in $j$ th assay in $k$ th generation, $g_{i j}$ is the (random) effect of $i$ th line in $j$ th memory assay, and $e_{i j k}$ is the residual. The effect of line $i$ in the two assays $\left(g_{i 1}, g_{i 2}\right)$ was assumed to follow bivariate normal distribution with covariance matrix G; no 


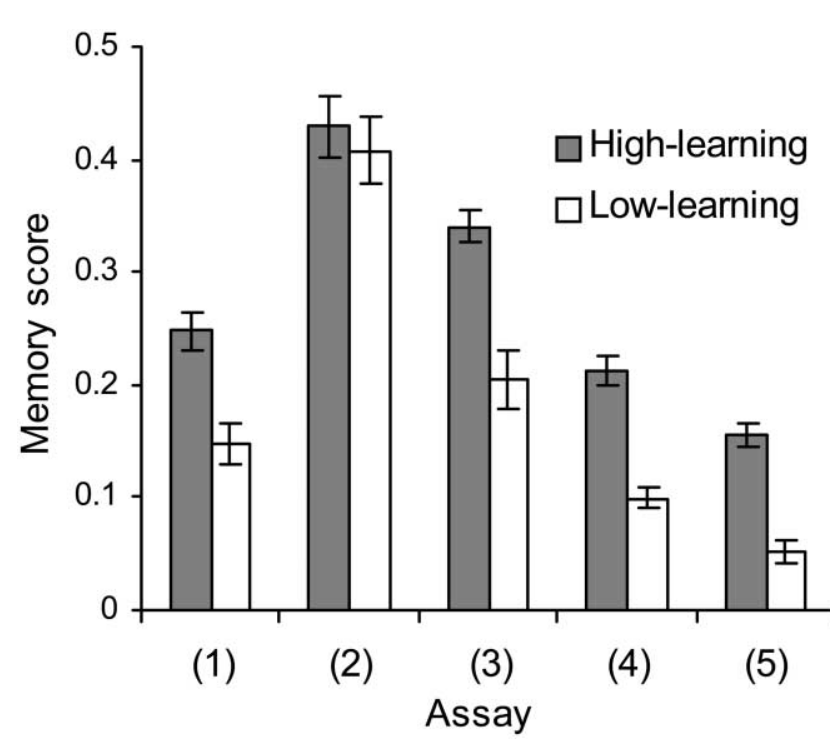

Figure 2. Memory scores (mean $\pm \mathrm{SE}$ ) of high-learning and low-learning lines in the five aversive conditioning assays described in the text. For assays 4 and 5, the results are based on data pooled over two experiments carried out six generations apart.

covariance between effects of different lines was assumed. The residuals were assumed to be normally distributed and uncorrelated, with different variances for the two memory assays. The model was fitted using restricted maximum likelihood (REML). The significance of the covariance of the line effects on the two memory assays was tested with the likelihood ratio test, by comparing -2 log likelihood of the above model with a restricted model in which the off-diagonal elements of $\mathbf{G}$ were set to 0 (Lynch and Walsh 1998). Because the number of replicates differed between the generations, and the line means $y_{i j k}$ were estimated with different confidence, we also carried out a weighted version of this analysis with the weights given by the inverse of the sampling variance (i.e., $\mathrm{SE}^{-2}$ ) of a given $y_{i j k}$.

\section{Unconditioned Response to Odors}

Differences in the learning performance might be confounded by differences in the response to odors independent of conditioning. We thus tested unconditioned response to the odors in the T-maze by offering groups of 50-70 naive (unconditioned) flies a choice between one of the odorants used in the memory assays $(0.6 \mathrm{~mL}$ octanol or $\mathrm{MCH}$ in $1 \mathrm{~L}$ paraffin $)$ and the odor of paraffin only. We used the same procedure as when testing for memory. The arcsine-transformed proportions of flies moving toward odorant rather than paraffin (excluding flies that remained in the central chamber of the T-maze) were analyzed with a nested ANOVA using the same model as that used for analysis of memory scores.

\section{Results}

\section{Comparison of High- and Low-Learning Lines}

The results of the five memory assays based on aversive conditioning are summarized in Figure 2 and Table 1. Twenty minutes after a single conditioning cycle, the high-learning lines showed a significantly better memory score than the low-learning lines (Fig. 2, assay 1). In contrast, the response measured 20 min after three massed conditioning cycles was similar in the two sets of lines (Fig. 2, assay 2).

Two and a half hours after five massed conditioning cycles the memory score was higher for high-learning than for lowlearning lines (Fig. 2, assay 3). This response reflected the combined effect of labile memory (presumably MTM) and ARM. When labile memory was erased with cold shock $30 \mathrm{~min}$ before testing, the performance index of both sets of lines was strongly reduced, but the difference between the high-learning and lowlearning lines remained (Fig. 2, assay 4). This implies that the high-learning lines showed a better ARM than the low-learning lines.

Twenty-four hours after five conditioning cycles in the spaced protocol, the high-learning lines again showed a much stronger response to conditioning than the low-learning lines (Fig. 2, assay 5). This suggests that the high-learning lines have better LTM. No significant variation among the lines within selection regimes was detected in any of the assays (lowest $P=0.18$ ).

\section{Relationship between Anesthesia-Resistant Memory and Long-Term Memory}

Inspection of the means of individual high-learning lines revealed a negative correlation between the memory scores in assay 4 (which is based on ARM) and assay 5 (which is thought to reflect largely LTM). This correlation was consistently observed in two experiments carried out at generations 100 (Fig. $3 a ; r=-0.75, P=0.052$ ) and 106 (Fig. $3 b ; r=-0.89$, $P=0.007)$. In particular, high-learning line 1 consistently performed worst in assay 4 and best in assay 5, whereas high-learning line 5 did best in assay 4 and worst in assay 5 , followed by high-learning line 2 . This among-line correlation was confirmed by the mixed-model analysis of the data from the two generations (weighted REML analysis: joint estimate $r=-0.90$; likelihood ratio test: $\chi^{2}=8.1$, $\mathrm{df}=1$, $P=0.005$; unweighted analysis yielded an even lower $P$ value). This result indicates a negative genetic correlation between performance in the two assays. The significance of this correlation is somewhat undermined by the fact that we did not detect significant variation among the high-learning lines for scores in either of the two assays (assay 4: $F_{6,76}=2.2, P=0.052$; assay 5: $\left.F_{6,77}=1.4, P=0.24\right)$. However, a multivariate ANOVA (MANOVA) on the means of those two scores calculated for high-learning lines separately for generations 100 and 106 indicated significant variation among lines (Wilks's $\lambda=0.017$, 
Table 1: Summary and statistical analysis of the five aversive learning assays

\begin{tabular}{llllll}
\hline & Assay 1 & Assay 2 & Assay 3 & Assay 4 & Assay 5 \\
\hline Number of conditioning cycles & 1 & 3 massed & 5 massed & 5 massed & 5 spaced \\
Conditioning-test interval & $20 \mathrm{~min}$ & $20 \mathrm{~min}$ & $2.5 \mathrm{~h}$ & $2.5 \mathrm{~h}$ & $24 \mathrm{~h}$ \\
Cold shock & No & No & No & Yes & No \\
$F$ (selection regime) & 16.5 & .2 & 22.9 & 37.7 & 40.4 \\
$P$ (selection regime) & .0023 & .67 & .0007 & $<.0001$ & $<.0001$ \\
Replicates per line & 4 & 3 & 4 & $11-13$ & $12-14$ \\
\hline
\end{tabular}

Note. $F$ statistics and $P$ values refer to the difference between the selection regimes; $\mathrm{df}=1,10$ for all assays. $N$ refers to the number of replicate memory scores per selection line. For assays 4 and 5, the analysis reported was done on pooled data from generations 100 and 106; the effect of selection regime was also highly significant $(P<0.001)$ for both assays when the two generations were analyzed separately.

$\left.F_{12,10}=5.6, P=0.005\right)$. No consistent differences were observed among the low-learning lines.

\section{Unconditioned Response to Odors}

When given a choice between an odorant and paraffin without prior conditioning, the flies from all lines preferred paraffin over both odorants (Fig. 4). No differences between the means of the two sets of lines were detected $\left(\mathrm{MCH}: F_{1,10.3}=0.23\right.$, $P=0.64$; octanol: $F_{1,10.0}=0.08, P=0.78$; three to four replicates per line and odorant tested on $2 \mathrm{~d}$ ). This - and the fact that both sets of lines showed the same memory score shortly after multiple conditioning cycles (assay 2 in Fig. 2) -indicates that the differences observed in other memory assays are not due to different responsiveness to the odors.

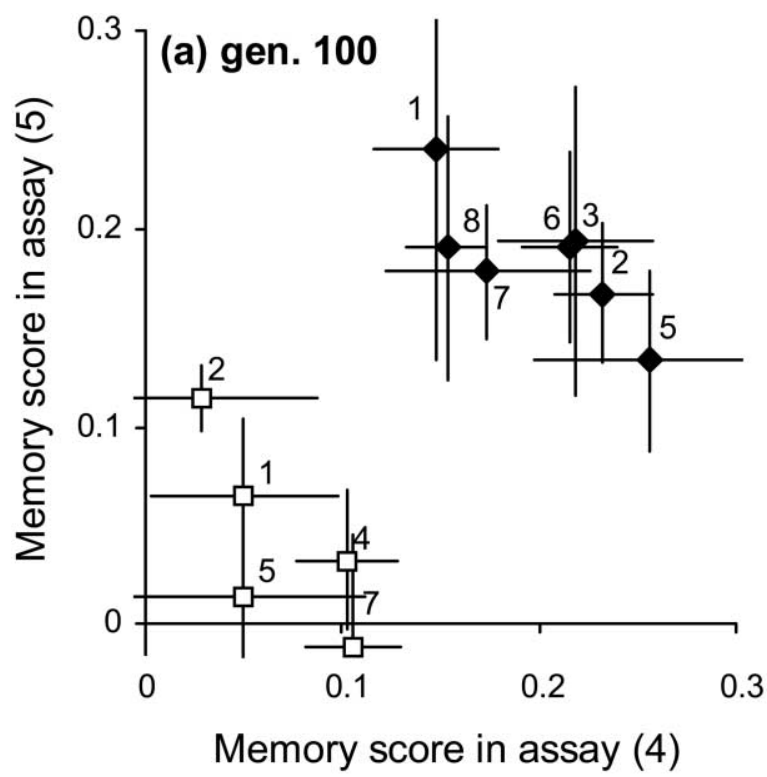

\section{Discussion}

The high-learning fly lines had been subject to selection for the ability to avoid an oviposition medium previously associated with quinine. Here we show that they are also better than the unselected low-learning lines at avoiding an odor previously associated with mechanical shock. Thus, their improved learning ability generalizes to a task involving another aversive stimulus (shock rather than bitter taste) and another behavioral context (movement in a T-maze rather than oviposition). This demonstrates that the response to selection was not based on enhanced salience of the particular stimuli used in the selection regime (Rescorla 1988). Thus, the response to selection in those lines was largely or entirely based on genetic variation affecting general processes of olfactory aversive learning rather than taskspecific aspects.

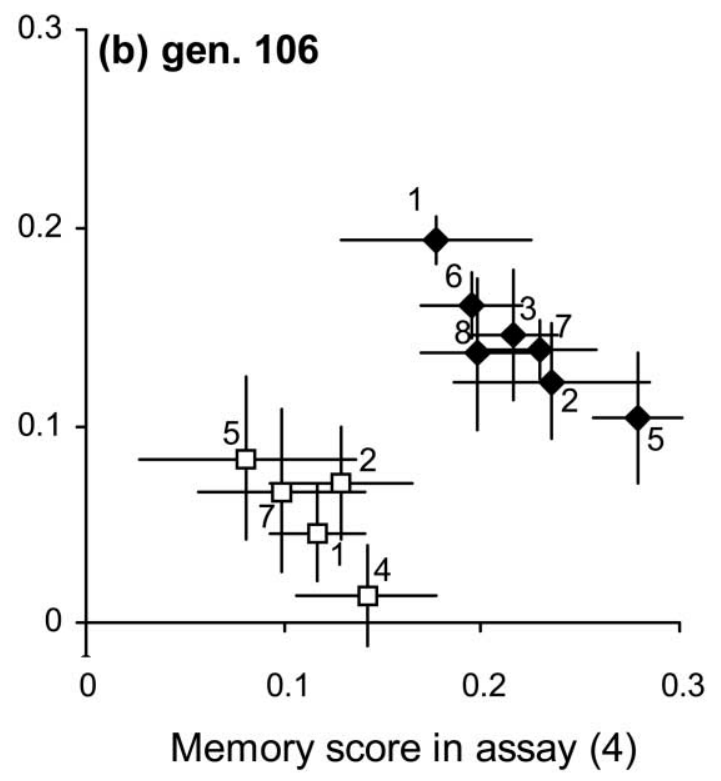

Figure 3. Relationship between memory scores (means \pm SE) in assays 4 and 5 across individual high-learning (filled diamonds) and lowlearning (open squares) lines. a, Generation 100; four replicates per line and assay. $b$, Generation 106, eight to 10 replicates per line and assay. Numbers next to symbols indicate line identity. 


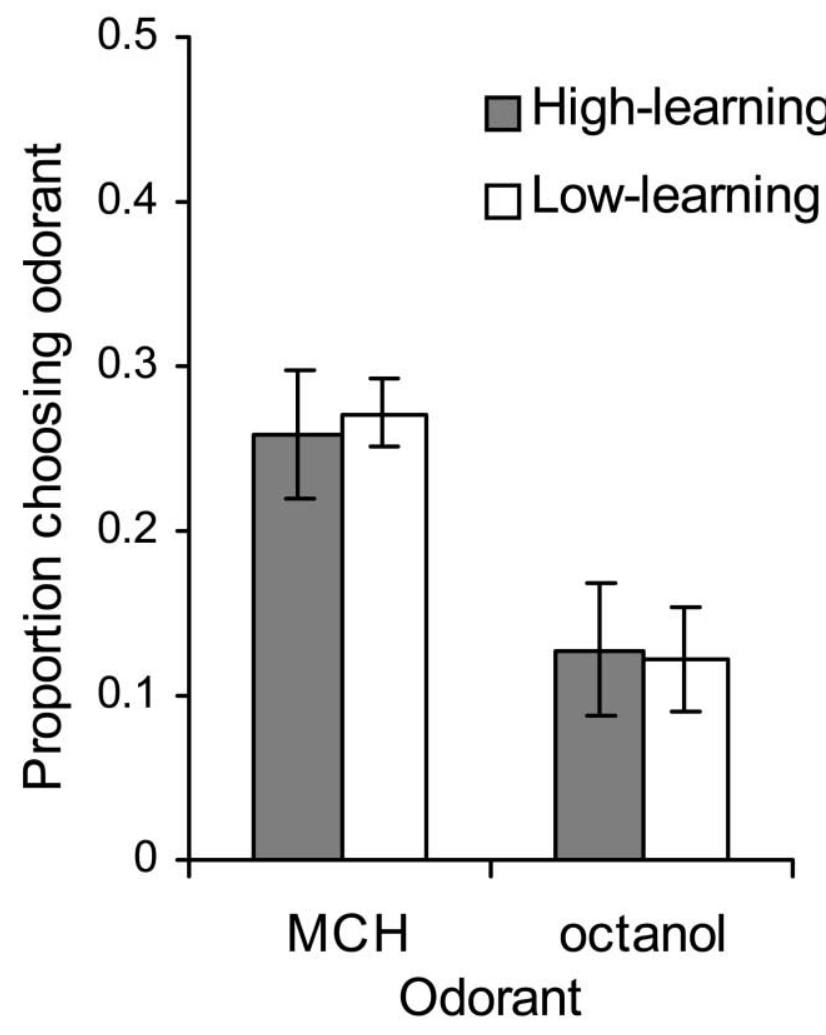

Figure 4. Unconditioned responses of the high- and low-learning lines to the odorants used in the memory assays. The graph shows the proportion (means $\pm \mathrm{SE}$ ) of flies choosing an odorant (octanol or methylcyclohexanol in paraffin) over the odor of paraffin only.

The five assays involving the olfactory aversive conditioning offer insights into the differences in dynamics of learning and consolidated memory. Comparison of assays 1 and 2 indicates that the high-learning lines learn faster than the low-learning lines, but the difference in 20-min response (presumably mostly based on STM) tends to disappear with multiple cycles of conditioning. This result parallels earlier results from the oviposition learning test (Fig. 5 in Mery and Kawecki 2002). Nonetheless, $2.5 \mathrm{~h}$ after several consecutive cycles of conditioning, the high-learning lines again show considerably better memory scores than the low-learning lines (assay 3 in Fig. 2). Thus, the memory trace is stronger in the high-learning lines also after multiple cycles of conditioning, even when it does not translate into better 20-min memory scores. A similar pattern was observed by Mery and Kawecki (2002) in the oviposition learning protocol, and they interpreted it as a slower decline of the memory trace in the high-learning lines. However, the comparison of assays 3 and 4 (Fig. 2) indicates that the difference in memory scores several hours after repeated conditioning reflects mostly or entirely greater buildup of ARM by the highlearning lines. The difference between assays 3 and 4 reflects the labile component of 2.5 -h memory (MTM), which was erased by cold shock in assay 4 . This difference is similar for the high- and low-learning lines, suggesting that the labile part of memory decays at a similar rate in those two sets of lines.

Previous work (e.g., Tully et al. 1994; Pascual and Preat 2001; Mery and Kawecki 2005) indicates that learned avoidance of odors shown by Drosophila $24 \mathrm{~h}$ after a spaced classical conditioning protocol is mostly based on LTM. Thus, the better performance of the high-learning lines in assay 5 (Fig. 2) indicates that they also have evolved improved LTM. It is possible that the pattern of exposure to conditioning under the selection regime (which was not controlled and which depended on the fly behavior) involved repeated exposure at intervals and that LTM is more easily formed under these conditions. Alternatively, improved LTM may be a by-product of improvements in the other aspects of learning.

This last hypothesis is made less plausible by the negative correlation between memory scores in assays 4 and 5 across the seven high-learning lines (Fig. 3). LTM does not form in a massed protocol such as that used in assay 4, so this correlation suggests a negative relationship between the improvements in the two forms of consolidated memory, ARM and LTM. Apparently, the high-learning lines with particularly good LTM show only a weak improvement in ARM (compared with unselected low-learning lines) and vice versa. One could argue that under the selection regime, under which these lines had evolved, improvement of both forms of consolidated memory would be superfluous. Optimal learned response within $6 \mathrm{~h}$ of conditioning might be achieved based on only one form of consolidated memory. However, at the time of those assays, the high-learning lines were still laying a substantial fraction of their eggs on the "wrong" substrate under the selection regime (F. Mery and T. J. Kawecki, unpublished data). Further improvement of the response would thus still be favorable, and so selection should continue to favor improvements in both ARM and LTM. Alternatively, the pattern in Figure 3 might result from differential inbreeding of the high-learning lines. It is difficult, however, to imagine why the effects of inbreeding on the two forms of consolidated memory should be negatively correlated. Furthermore, previous assays on crosses between replicate high-learning lines showed no evidence of inbreeding depression for learning performance (Kawecki and Mery 2006), larval competitive ability (Mery and Kawecki 2003), or fecundity (Kawecki and Mery 2006).

We think it is likely that the apparent negative correlation between ARM and LTM reflects a trade-off between these two memory forms rather than a lack of selection or inbreeding. Such a trade-off would be consistent with the hypothesis that the mechanisms underlying these forms of consolidated memory are, to some degree, antagonistic (Isabel et al. 2004). Whatever the reason for the covariation of the high-learning lines along the ARM-LTM axis in Figure 3, it suggests that the highlearning lines have to some degree diverged from one another (see also Kawecki and Mery 2006). 
The high-learning lines were subject to selection for improved learning performance under an ecologically relevant selection regime. The results reported here suggest that the genetic variation underlying the response to selection affected several aspects of learning and memory formation, occurring both early in the process (the learning rate) and late, up to several hours after the stimuli to be associated were perceived (ARM and LTM). It remains to be seen whether these diverse improvements reflect a single underlying mechanism due to the same allele substitutions or whether they are genetically independent and evolved in concert only because all of them were simultaneously favored by the selection regime.

\section{Acknowledgments}

This work was supported by grants from the Swiss National Science Foundation (3100A0-100387) to T.J.K. and from the Ministère de la Recherche (Agence Nationale de la Recherche Neurosciences) to T.P.

\section{Literature Cited}

Davis R.L. 2005. Olfactory memory formation in Drosophila: from molecular to systems neuroscience. Annu Rev Neurosci 28:275-302.

Dezazzo J. and T. Tully. 1995. Dissection of memory formation: from behavioral pharmacology to molecular genetics. Trends Neurosci 18:212-218.

Dubnau J. and T. Tully. 1998. Gene discovery in Drosophila: new insights for learning and memory. Annu Rev Neurosci 21:407-444.

Dukas R. 2004. Evolutionary biology of animal cognition. Annu Rev Ecol Evol Syst 35:347-374.

Healy S.D. and V.A. Braithwaite. 2000. Cognitive ecology: a field of substance? Trends Ecol Evol 15:22-26.

Isabel G., A. Pascual, and T. Preat. 2004. Exclusive consolidated memory phases in Drosophila. Science 304:1024-1027.

Kawecki T.J. and F. Mery. 2006. Genetically idiosyncratic re- sponses of Drosophila melanogaster populations to selection for improved learning ability. J Evol Biol 19:1265-1274.

Littell R.C., G.A. Milliken, W.W. Stroup, and R.D. Wolfinger. 1996. SAS System for Mixed Models. SAS Institute, Cary, NC.

Lynch M. and B. Walsh. 1998. Genetics and Analysis of Quantitative Traits. Sinauer, Sunderland, MA.

Macphail E.M. and J.J. Bolhuis. 2001. The evolution of intelligence: adaptive specialization versus general process. Biol Rev 76:341-364.

Margulies C., T. Tully, and J. Dubnau. 2005. Deconstructing memory in Drosophila. Curr Biol 15:R700-R713.

Menzel R. 1999. Memory dynamics in the honeybee. J Comp Physiol A 185:323-340.

Mery F. and T.J. Kawecki. 2002. Experimental evolution of learning ability in fruit flies. Proc Natl Acad Sci USA 99: 14274-14279.

- 2003. A fitness cost of learning ability in Drosophila melanogaster. Proc R Soc B 270:2465-2469.

- 2004. An operating cost of learning in Drosophila melanogaster. Anim Behav 68:589-598.

- 2005. A cost of long-term memory in Drosophila. Science 308:1148.

Pascual A. and T. Preat. 2001. Localization of long-term memory within the Drosophila mushroom body. Science 294: 1115-1117.

Rescorla R.A. 1988. Behavioral studies of Pavlovian conditioning. Annu Rev Neurosci 11:329-352.

SAS Institute. 1989. SAS/STAT User's Guide. Version 6, 4th ed. SAS Institute, Cary, NC.

Sokal R.R. and F.J. Rohlf. 1995. Biometry: The Principles and Practice of Statistics in Biological Research. 3rd ed. W.H. Freeman, New York.

Tully T., T. Preat, S.C. Boynton, and M. Del Vecchio. 1994. Genetic dissection of consolidated memory in Drosophila. Cell 79:35-47.

Wadell S. and W.G. Quinn. 2001. Flies, genes, and learning. Annu Rev Neurosci 24:1283-1309. 